\title{
RADIAÇÃO SOLAR GLOBAL ESTIMADA A PARTIR DA INSOLAÇÃO PARA MACAPÁ (AP)
}

\author{
LIANA PEREIRA BELÚCIO ${ }^{1}$, ANA PAULA NUNES DA SILVA ${ }^{1}$, LEANDRO RODRIGUES \\ SOUZA $^{2}$, GEBER BARBOSA DE ALBUQUERQUE MOURA ${ }^{3}$
}

\author{
${ }^{1}$ Universidade do Estado do Amapá (UEAP), Colegiado de Engenharia Ambiental, Macapá, AP, Brasil \\ ${ }^{2}$ Universidade Federal do Amapá (UFAP), Departamento de Física, Macapá, AP, Brasil \\ ${ }^{3}$ Universidade Federal Rural de Pernambuco (UFRPE), Departamento de Agronomia, Recife, PE, Brasil \\ lianabelucio@hotmail.com,apns@ymail.com,souzalr@msn.com,geber@depa.ufpe.edu.br
}

Recebido Setembro de 2013 - Aceito Junho de 2014

\begin{abstract}
RESUMO
O presente trabalho tem por objetivo aplicar a análise de regressão para estimar a radiação solar global em função da insolação na cidade de Macapá (AP), sendo esta abordagem uma alternativa às estações meteorológicas sem registros de radiação solar global. Utilizou-se dados diários para os doze meses do ano, no período 2006-2012, de radiação solar global observadas na Plataforma de Coleta de Dados (PCD) do Instituto Nacional de Pesquisas Espaciais (INPE) $\left(0,04^{\circ} \mathrm{N} ; 51,08^{\circ} \mathrm{W}\right.$; $16,0 \mathrm{~m}$ ) e de insolação provenientes da estação convencional do Instituto Nacional de Meteorologia (INMET) $\left(0,05^{\circ} \mathrm{S} ; 51,12^{\circ} \mathrm{W} ; 14,5 \mathrm{~m}\right)$. A estimativa da radiação solar global a partir da insolação foi realizada utilizando-se as equações de regressão linear simples e o modelo de Angström-Prescott. Os coeficientes de correlação de Pearson (r) mensais analisados revelaram-se satisfatórios $(0,76$; 0,82 ), e os maiores valores mensais dos coeficientes angulares "b" da equação Angström-Prescott foram observados entre os meses chuvosos (verão) $(b=0,45 ; 0,44)$, o que indicou que a radiação solar global difusa é maior nesse período do ano, do que nos meses menos chuvosos, provavelmente devido à nebulosidade e aerossóis presentes na atmosfera. Portanto, como é alta a relação entre os elementos meteorológicos estudados, pode-se utilizar os dados de radiação global estimados a partir dos registros de insolação para Macapá (AP).

Palavras-chave: fotoperíodo, razão de radiação solar global, razão de insolação.
\end{abstract}

\begin{abstract}
GLOBAL SOLAR RADIATION ESTIMATION FROM INSOLATION DATA FOR MACAPÁ CITY (AP)

The aim of this study was to applied the regression analysis results in order to estimate the solar global radiation as a function of the insolation at Macapa City (AP), this being an alternative to the weather stations that has no records of the global solar radiation. Daily data for the twelve months of the year were used, in the period 2006-2012, of radiation observed in Data Collection Platform (PCD) of the Instituto Nacional de Pesquisas Espaciais (INPE) and insolation from the conventional station of the Instituto Nacional de Meteorologia (INMET) from Brazil. The estimation of global solar radiation with insolation was performed using simple linear regression equations and the Angström-Prescott model. The monthly Pearson correlation coefficients ( $\mathrm{r}$ ) analyzed proved satisfactory (0.76 and 0.82 ); the highest monthly values of the coefficients " $b$ " of the Angström-Prescott equation were found in the rainy season (summer) $(b=0.45 ; 0.44)$, indicating that the diffuse solar radiation is higher at this time of year than during the driest months, probably due to aerosols and clouds in the atmosphere. Therefore, with the high ratio between this meteorological elements studied, the global radiation estimated from the insolation can be used for Macapá (AP).
\end{abstract}

Keywords: photoperiod, solar radiation ratio, insolation ratio. 


\section{INTRODUÇÃO}

A radiação solar é um elemento meteorológico que influencia processos desde a escala micrometeorológica, quando se tratados fluxos turbulentos, até a grande escala, ao promover aquecimento diferencial no planeta e gerando a circulação geral da atmosfera. No atual contexto de desenvolvimento sustentável, a humanidade busca fontes alternativas de energia, sendo a energia solar uma destas fontes. Portanto, estudos que relacionados a esta variável são importantes para a avaliação de possíveis regiões com potencial para usar este tipo de energia, assim como, se avaliar como a radiação solar influencia os fluxos de energia em superfície.

De acordo com Borges et al. (2010) a radiação solar é a força motriz para muitos processos físico-químicos e biológicos que ocorrem no sistema Superfície-Atmosfera, e constitui-se em importante variável meteorológica em estudos de necessidade hídrica de culturas, modelagem do crescimento e produção vegetal, mudanças climáticas, entre outros.

A radiação solar incidente sobre a superfície é fator determinante e condiciona os processos que ocorrem nesse ambiente, interferindo diretamente em diversos ciclos biogeoquímicos (água, carbono, nitrogênio, fosforo, enxofre, entre outros). Assim, a variação da quantidade de radiação solar incidente, resulta em condições ambientais diferenciadas e, por conseguinte, promove alteração nos demais processos (Araújo, 2005). Assim a análise e determinação da radiação solar no topo da atmosfera (radiação solar extraterrestre) são essenciais, uma vez que, através da comparação com a radiação na superfície, consegue-se caracterizar a qualidade atmosférica com relação a sua turbidez (Querino et al., 2011).

Segundo Almorox et al. (2008), informações locais da radiação solar global, além de ser utilizadas em simulações de crescimento e produtividade das culturas agrícolas podem ser, também, aplicadas para dimensionamento de sistemas alternativos de produção de energia. Ainda de acordo com os autores, os coeficientes de regressão linear dependem da latitude, época do ano e altitude, e variam em função das mudanças no tipo e espessura de nuvens e concentração de poluentes. A quantidade de radiação solar global e sua distribuição temporal são as principais variáveis para a concepção de sistemas de energia solar (Celik e Yorukoglu, 2006).

Para localidades com ausência de observações de radiação solar, valores estimados podem ser obtidos através de modelos matemáticos, que diferem entre si pelo grau de complexidade e pelas variáveis de entrada. Os modelos de Angström (Angström, 1924) e de Johnson-Woodward (Rivington et al., 2005; Miller et al., 2008) utilizam a insolação (horas de brilho solar fornecida pelo heliógrafo) para estimar a radiação solar incidente.
Segundo Liu et al. (2009) há poucas informações que comparem os coeficientes de Angstrom-Prescott calibrados localmente com os recomendados pela FAO, e seus efeitos na estimativa da radiação solar global. Este fato foi corroborado por Buriol et al. (2012) ao relatar que poucas estações meteorológicas possuem registros de radiação solar global, devido ao elevado custo e frequentes manutenções pelas quais os aparelhos utilizados precisam passar.

Em razão da significativa extensão territorial brasileira e dificuldade de acesso, somente em alguns poucos locais são encontrados dados consistentes sobre parâmetros meteorológicos. Tal deficiência de informações, que inclui a falta de dados sobre radiação solar na superfície, inviabiliza o uso no beneficiamento da produção de energia elétrica por sistemas fotovoltaicos, limitando seu uso para moradias isoladas e pequenas aplicações comunitárias (Marques et al., 2012).

Observações da radiação solar global geralmente são realizadas em Estações Meteorológicas Automáticas (EMA), já a insolação é registrada em Estações Meteorológicas Convencionais (EMC). Devido algumas localidades ter apenas EMC, nestas regiões os dados de insolação ainda são obtidos em detrimento de dados de radiação solar, e em muitos outros países a medida direta da radiação solar ainda é escassa quando comparada com as medições de temperatura do ar e precipitação. Esta situação reflete, provavelmente, não apenas o custo de aquisição do equipamento, mas também os problemas de manutenção e calibração dos equipamentos que medem a radiação solar (Liu et al., 2009; Robaa, 2009).

Partindo desta problemática, alguns trabalhos foram desenvolvidos com objetivo de desenvolver equações que estimem a radiação solar em função da insolação, a saber: Campelo Júnior (1998), para Santo Antônio do Leverger (MT); Dornelas et al. (2006), para Brasília (DF); Borges et al. (2010), para Cruz das Almas (BA); Pereira et al. (2010), para Pedra Azul (MG); Andrade Júnior et al. (2012) para Parnaíba (PI). Todas estas para regiões tropicais, onde a variabilidade sazonal da radiação solar global é baixa.

Deste modo, objetiva-se neste trabalho, ajustar os coeficientes a e b, para que se possa estimar a radiação solar global em função de valores de insolação utilizando dados diários e avaliar a distribuição anual e mensal da radiação solar global no período estudado.

\section{MATERIAL E MÉTODOS}

A cidade de Macapá, Estado do Amapá, encontra-se no ponto com latitude e longitude $0,03^{\circ} \mathrm{N}$ e $51,05^{\circ} \mathrm{W}$, respectivamente. De acordo com as normais climatológicas do Instituto Nacional de Meteorologia (INMET, 2009) a cidade de Macapá possui duas estações distintas relacionadas 
à precipitação: a estação chuvosa, de janeiro a junho, e, uma estação menos chuvosa, de julho a dezembro. De acordo com dados do período de 1968 a 2012 do INMET, a precipitação pluvial média varia de aproximadamente $330,5 \mathrm{~mm}$.mês ${ }^{-1}$ nos meses chuvosos, a 91,5 mm. mês $^{-1}$ nos demais meses (Figura 1A). A insolação, que representa o número de horas de brilho solar efetivo, os mínimos valores, em torno de 114 horas.mês ${ }^{-1}$, ocorrem em março, período de maior precipitação, enquanto que os máximos, aproximadamente 285 horas.mês ${ }^{-1}$, são registrados em outubro (Figura 1B).

Estas condições, observadas no Amapá, estão diretamente associadas à intensa radiação solar incidente na região Tropical, além da influência direta da Zona de Convergência Intertropical (ZCIT), que é o principal sistema meteorológico responsável pelo regime de chuvas na região amazônica (Horel et al., 1989).

Foram utilizados dados diários de radiação solar global (Qg) registrados por um radiômetro, com piranômetro tipo kipp e Zonen CM22 e Pyrgeometer tipo Kipp e Zonen CG4, de funcionamento eletrônico na Plataforma de Coleta de Dados (PCD), pertencente ao Instituto Nacional de Pesquisas Espaciais (INPE) localizada em Macapá $\left(0,04^{\circ} \mathrm{N} ; 51,08^{\circ} \mathrm{W}\right.$; $16,0 \mathrm{~m}$ ). Os dados diários de insolação (n) foram registrados por um heliógrafo, tipo Campbell-Stokes, instalado na estação meteorológica pertencente ao $1^{\circ}$ Distrito de Meteorologia $\left(1^{\circ}\right.$ DISME), do Instituto Nacional de Meteorologia (INMET), situada no Distrito de Fazendinha $\left(0,05^{\circ} \mathrm{S} ; 51,12^{\circ} \mathrm{W} ; 14,5 \mathrm{~m}\right)$ (Figura 2).

Os dados foram coletados do período de janeiro de 2006 a dezembro de 2012, em escala diária. O controle de qualidade dos dados seguiu o proposto por Persaud et al. (1997) e Moradi (2009), sendo removidos os dados: dias com ausência de insolação ou radiação solar; dias em que a razão de insolação (n; insolação/N; fotoperíodo) e a transmissividade atmosférica global (Qg; radiação solar global/Qo; radiação solar extraterrestre) para radiação solar, apresentaram valores maiores do que 1; e, valores de Qg superiores a 0,85Qo, pois supõem-se que a transmissividade de céu sem nebulosidade é no máximo $85 \%$. Deste modo, foram obtidos 2.290 dias com dados analisados, pois do total de dados observados (2.642), 13,3\% foram considerados inconsistentes, baseados nos critérios acima.

Determinou-se, para cada dia, a transmissividade atmosférica global (R) e a razão de insolação (h), com a utilização das Equações 1 e 2:

$$
\begin{aligned}
& R=\frac{Q g}{Q o} \\
& h=\frac{n}{N}
\end{aligned}
$$

em que: Qg, é a radiação solar global $\left(\mathrm{MJ} \mathrm{m}^{-2} \mathrm{~d}^{-1}\right)$; $\mathrm{Qo}$, a radiação solar extraterrestre $\left(\mathrm{MJ} \mathrm{m}^{-2} \mathrm{~d}^{-1}\right)$; n é a insolação $\left(\mathrm{h}^{\left.-\mathrm{d}^{-1}\right)}\right.$; $\mathrm{N}$ é a duração máxima do brilho solar ou fotoperíodo $\left(h \cdot \mathrm{d}^{-1}\right)$. Os valores de $\mathrm{N}$ e Qo foram calculados, respectivamente, pelas Equações 3 e 4:

$$
\begin{aligned}
& \mathrm{N}=2 \mathrm{H} / 15 \text { [horas }] \\
& Q_{o}=\frac{86400 \times S_{o}}{D^{2} \pi} \\
& \left(H \frac{\pi}{180} \operatorname{sen} \phi \times \operatorname{sen} \delta+\cos \phi \times \cos \delta \times \operatorname{sen} H\right) \quad\left[\mathrm{MJ} \cdot \mathrm{m}^{-2} \mathrm{~d}^{-1}\right]
\end{aligned}
$$

em que: $\phi$, é a latitude do local $\left({ }^{\circ}\right) ; \delta$, declinação solar $\left({ }^{\circ}\right)$, $\mathrm{D}$, distância média Terra-Sol (km), So, constante Solar; H, a duração do dia solar em radianos, calculado pela Equação 5 (Buriol et al., 2012):

$$
H=\arccos (-\operatorname{tg} \delta \times \operatorname{tg} \phi)+0,83^{\circ}
$$
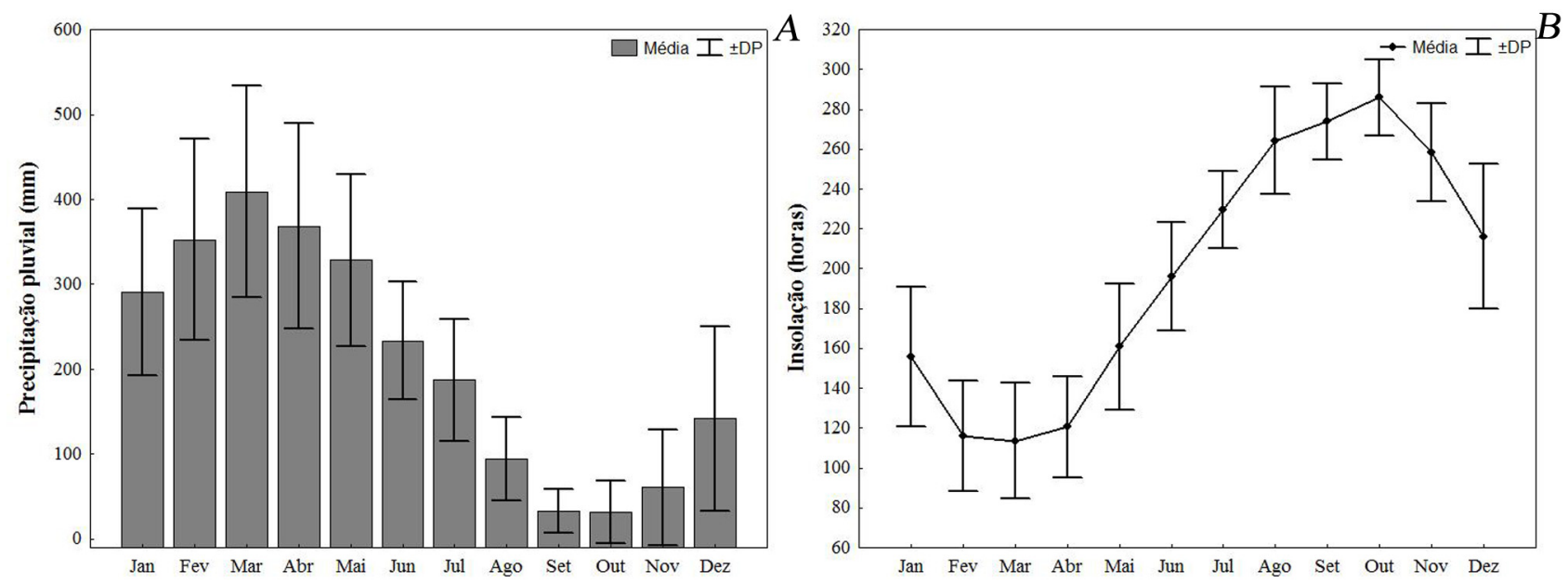

Figura 1 - Média e desvio padrão da precipitação pluvial (A) e insolação (B) climatológica em Macapá (AP). 


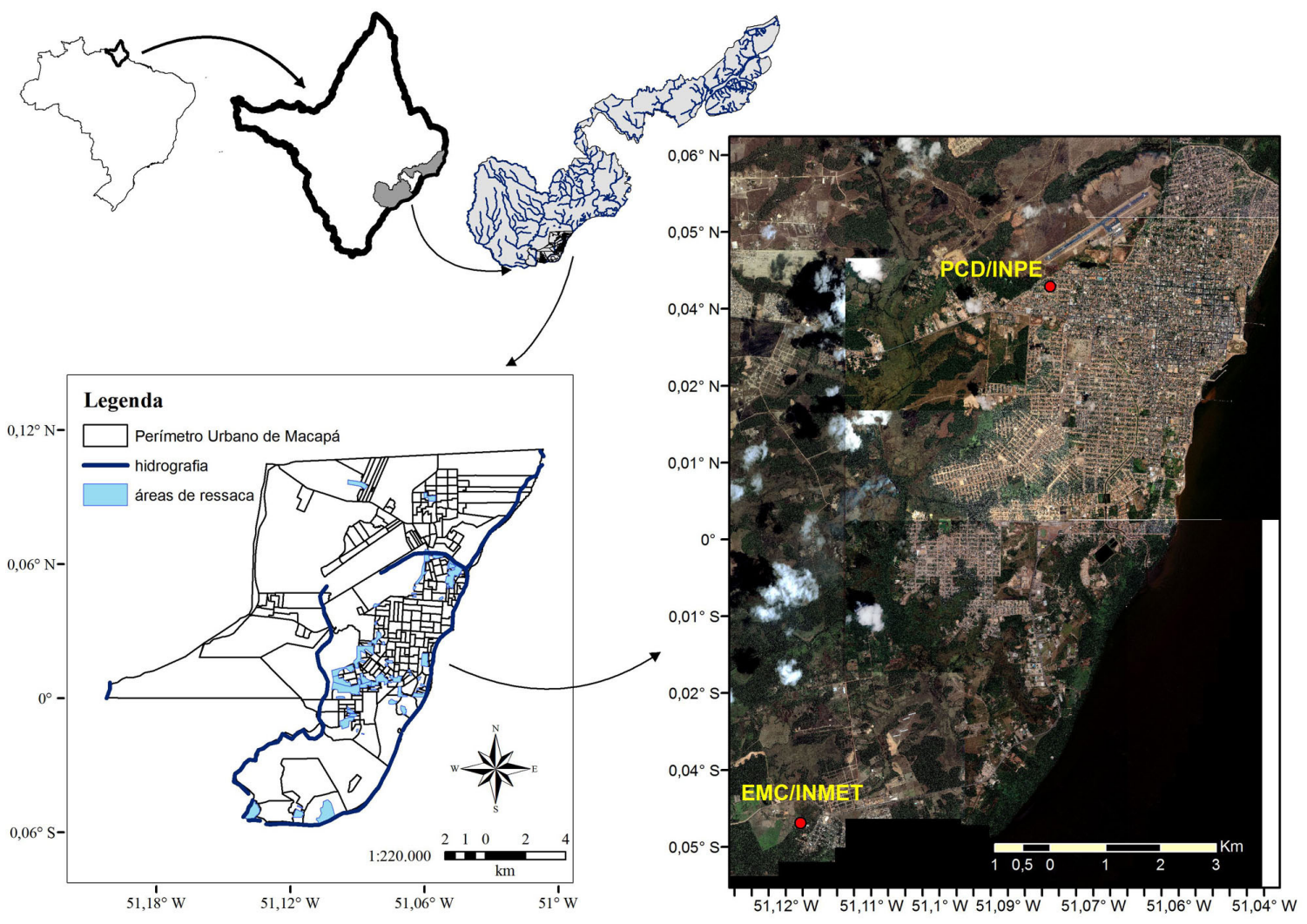

Figura 2 - Área em estudo.

Para a análise de regressão entre a radiação e insolação disponíveis na região em estudo, utilizou-se o software Statistica 9.0 (Statsoft, 2009). A estimativa da radiação solar global (Qg), a partir da insolação real (n), foi realizada utilizando-se a equação de regressão linear entre a razão de insolação e a transmitância atmosférica, como também o modelo de Angström-Prescott (Dantas et al., 2003), conforme Equação 6:

$$
Q g=Q o\left(a+b \frac{n}{N}\right)
$$

Como o sugerido por Dantas et al. (2003), por análise de regressão, obtiveram-se os coeficientes a e b, que caracterizam a transmitância atmosférica, sendo $a$, o coeficiente que expressa a fração da radiação solar extraterrestre, que atinge a Terra em dias totalmente nublados, correspondente à fração difusa; $b$ é o coeficiente complementar que expressa o total de radiação solar global.

Para verificar o grau de ajustamento, foram calculados os respectivos coeficientes de determinação e o grau da correlação foi verificado através da Tabela 1, proposta por Devore (2006), que indica o grau da correlação pelos coeficientes de Pearson.

Os valores reais de radiação solar global estimados pelos modelos propostos neste trabalho foram avaliados estatisticamente com os valores reais de radiação solar global observados entre janeiro e outubro de 2013 (período limite disponível no INMET) por meio do índice de concordância de Willmott (Willmott et al. 1985), d (Equação 7), o coeficiente de correlação de Pearson, r (Equação 8) e pelo coeficiente de confiança ou desempenho (Equação 9).

$$
d=1-\left[\frac{\sum_{i=1}^{n}\left(E_{i}-O_{i}\right)^{2}}{\sum_{i=1}^{n}\left(\left|E_{i}-\bar{O}\right|+\left|O_{i}-\bar{O}\right|\right)^{2}}\right]
$$

em que: $d$, é o índice de exatidão, adimensional (seus valores podem variar de 0 , para nenhuma concordância, a 1 , para uma

Tabela 1 - Coeficiente de correção estatística, ou coeficiente de correção de Pearson.

\begin{tabular}{ll}
\hline $\mathbf{r}$ & Definição \\
\hline 0,00 a 0,19 & Correlação bem fraca \\
0,20 a 0,39 & Correlação fraca \\
0,40 a 0,69 & Correlação moderada \\
0,70 a 0,89 & Correlação forte \\
0,90 a 1,00 & Correlação muito forte \\
\hline Fonte: Devore (2006)
\end{tabular}


concordância perfeita); $E_{i}$, o valor estimado; $O_{i}$, é o valor observado; $\bar{O}$, é a média dos valores observados; e $N$, o número de observações.

$$
r=\frac{\sum_{i=1}^{n}\left(x_{i}-\bar{x}\right) \times\left(y_{i}-\bar{y}\right)}{\sqrt{\sum_{i=1}^{n}\left(x_{i}-\bar{x}\right)^{2}} \times \sqrt{\sum_{i=1}^{n}\left(y_{i}-\bar{y}\right)^{2}}}
$$

em que: $r$, é o coeficiente de correlação de Pearson, adimensional; $x_{1}, x_{2}, \ldots, x_{n}$ e $y_{1}, y_{2}, \ldots, y_{n}$, os valores medidos de ambas as variáveis,

$$
c=r \times d
$$

em que: $c$, é o coeficiente de confiança ou desempenho, adimensional; $r$, o coeficiente de correlação de Pearson; e $d$, o índice de concordância de Willmott.

O coeficiente $c$, proposto por Camargo e Sentelhas (1997), é interpretado de acordo com a Tabela 2.

Separaram-se os dias analisados pelo critério de presença de céu sem nebulosidade e nublado, tendo como base a razão entre a radiação solar global e a radiação solar extraterrestre, esta razão é denominada como transmitância atmosférica. Utilizando-se da metodologia empregada por Culf et al. (1995) e Galvão e Fisch (2000) para o estudo da disponibilidade de energia solar na região Amazônica, os autores concluíram que os dias que apresentaram transmitância com valores inferior a 0,35 podem ser considerados nublados, e aqueles em que os valores foram maiores do que 0,50 podem ser considerados como dia de céu sem nebulosidade.

\section{RESULTADOS E DISCUSSÃO}

A radiação solar extraterrestre apresentou pouca variação entre o período de estudo, sendo seu valor médio, para o

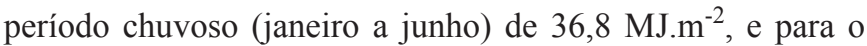

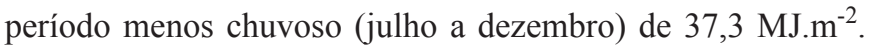
A pouca variação nos valores se deve a proximidade da área de estudo da linha do Equador, já que esta variável depende apenas do cosseno da latitude do local e da declinação do Sol.

Tabela 2 - Critérios de interpretação do índice de desempenho (c) dos métodos de estimativa de Qg.

\begin{tabular}{ll}
\hline c & Classificação \\
\hline$>0,85$ & Ótimo \\
$0,76-0,85$ & Muito Bom \\
$0,66-0,75$ & Bom \\
$0,61-0,65$ & Mediano \\
$0,51-0,60$ & Sofrível \\
$0,41-0,50$ & Mal \\
$<=0,40$ & Péssimo \\
\hline
\end{tabular}

Como a primeira apresenta valor próximo ao nulo, a radiação solar extraterrestre apresentou valores semelhantes ao longo do ano. Já os valores de insolação total mensal variaram entre 150,97 horas (período chuvoso) e 238,04 horas (período menos chuvoso).

Os valores extremos de radiação solar global (máximo e mínimo), no período chuvoso foram encontrados no mês de abril, com 32,0 MJ.m ${ }^{-2}$ e 6,0 MJ.m ${ }^{-2}$ respectivamente, com média de $16,11 \mathrm{MJ} . \mathrm{m}^{-2}$. Já para a estação menos chuvosa (julho a dezembro) foram encontrados os valores mais elevados de

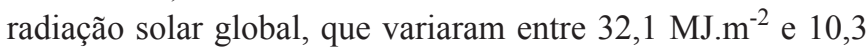
MJ.m ${ }^{-2}$, tendo em vista que a ZCIT não atua nesta época, o que facilita a passagem de mais radiação devido à diminuição de nebulosidade. Essa baixa variabilidade sazonal da radiação global evidencia a influência do movimento de translação da Terra sobre esta região, devido a esse movimento no local de análise, os valores são semelhantes durante o ano todo.

Resultados semelhantes foram encontrados por Aguiar et al. (2011) para valores de radiação solar global em dois sítios experimentais no Pará (pastagem e floresta), que apresentaram valores que oscilaram em torno de $38 \mathrm{MJ} . \mathrm{m}^{-2}$ (estação úmida) e 35 MJ.m² (estação chuvosa) em ambas as áreas. Em Macapá estas diferenças não apresentaram grande variabilidade devido à região não apresentar estações de seca e chuva características, mas sim uma estação com chuvas excedentes e outra com chuvas moderadas.

Nas Figuras 3 e 4 estão representados os diagramas de dispersão dos valores diários da razão de insolação e da transmitância atmosférica global (Qg/Qa) entre 2006 e 2012 para os doze meses do ano para Macapá (AP). Constatou-se para todos os meses, elevada correlação entre os valores diários da razão de insolação e da transmitância atmosférica global. Fato confirmado pelos resultados das equações de regressão, em que apresentaram coeficientes de correlação considerados forte, de acordo com classificação proposta por Devore (2006), com 0, 82 em janeiro e maio.

Conforme Buriol et al. (2012) os valores que aparecem abaixo da reta representam situações em que o sol brilhou somente nas primeiras e/ou nas últimas horas do dia, quando a radiação solar global é menor. Os valores acima da linha de regressão advêm de casos em que houve brilho solar somente nas horas próximas ao meio dia, quando a radiação solar global é mais intensa.

Para os meses do período chuvoso, que de acordo com o INMET (2009) possui nebulosidade mensal de 0,9 em Macapá, os coeficientes de correlação (r) variaram de 0,82 (janeiro) a 0,72 (abril) (Tabela 3). Estes resultados indicaram haver correlação forte entre os dois elementos meteorológicos, transmitância atmosférica e razão de insolação, na estação chuvosa. 

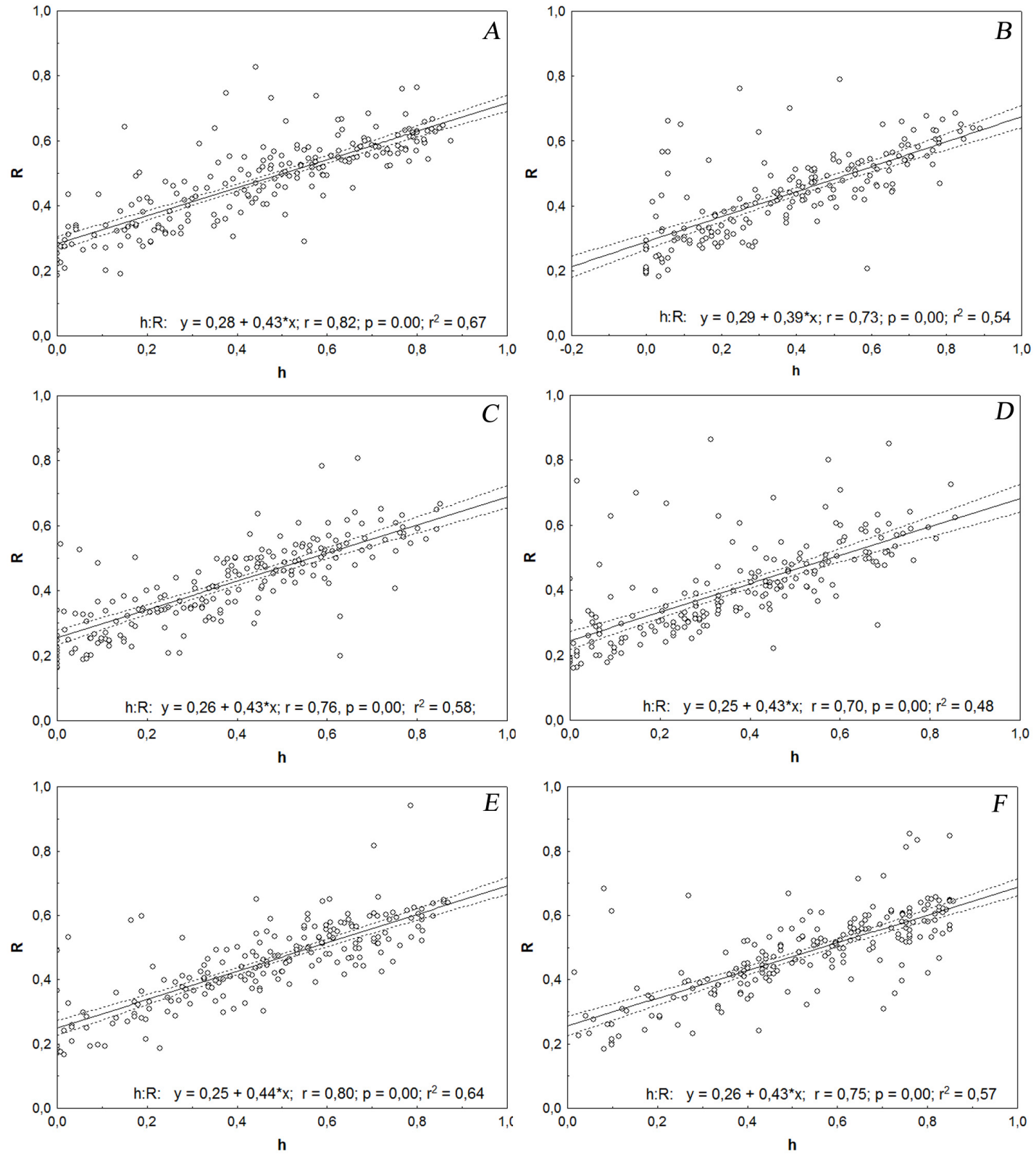

Figura 3 - Relação entre os valores diários de razão de radiação solar global (R) e razão de insolação (h) para os meses de janeiro (A), fevereiro (B), março (C), abril (D), maio (E) e junho (F), no período de 2006 a 2012, em Macapá (AP).

De julho a dezembro, meses compreendidos no período menos chuvoso, que possui nebulosidade mensal de 0,7 (INMET, 2009), os coeficientes de correlação (r) variaram de 0,48 (setembro) a 0,74 (dezembro) (Tabela 3). Estes resultados mostraram associação moderada para os meses entre esses dois elementos meteorológicos. O mês que apresentou maior coeficiente de correlação foi dezembro com valor de 0,74 , já setembro apresentou menor coeficiente de determinação $(0,23)$, sendo este o mês menos chuvoso na cidade.

Nos meses do período chuvoso os coeficientes lineares das equações de regressão variaram entre 0,24 e 0,29 , enquanto que nos meses do período menos chuvoso oscilaram entre 0,31 e 0,40 . Como estes valores estão relacionados com a radiação solar difusa, indicam que apesar de no período chuvoso haver altos índices de nebulosidade, é no período menos chuvoso que há maior dispersão de radiação solar em Macapá. Este fato ocorre devido aos baixos índices de precipitação e à grande concentração de material particulado devido ao aumento de queimadas na região.

Os coeficientes angulares das equações propostas estão relacionados à radiação solar global direta, que depende da declinação solar e da transmissividade da atmosfera. Nos 

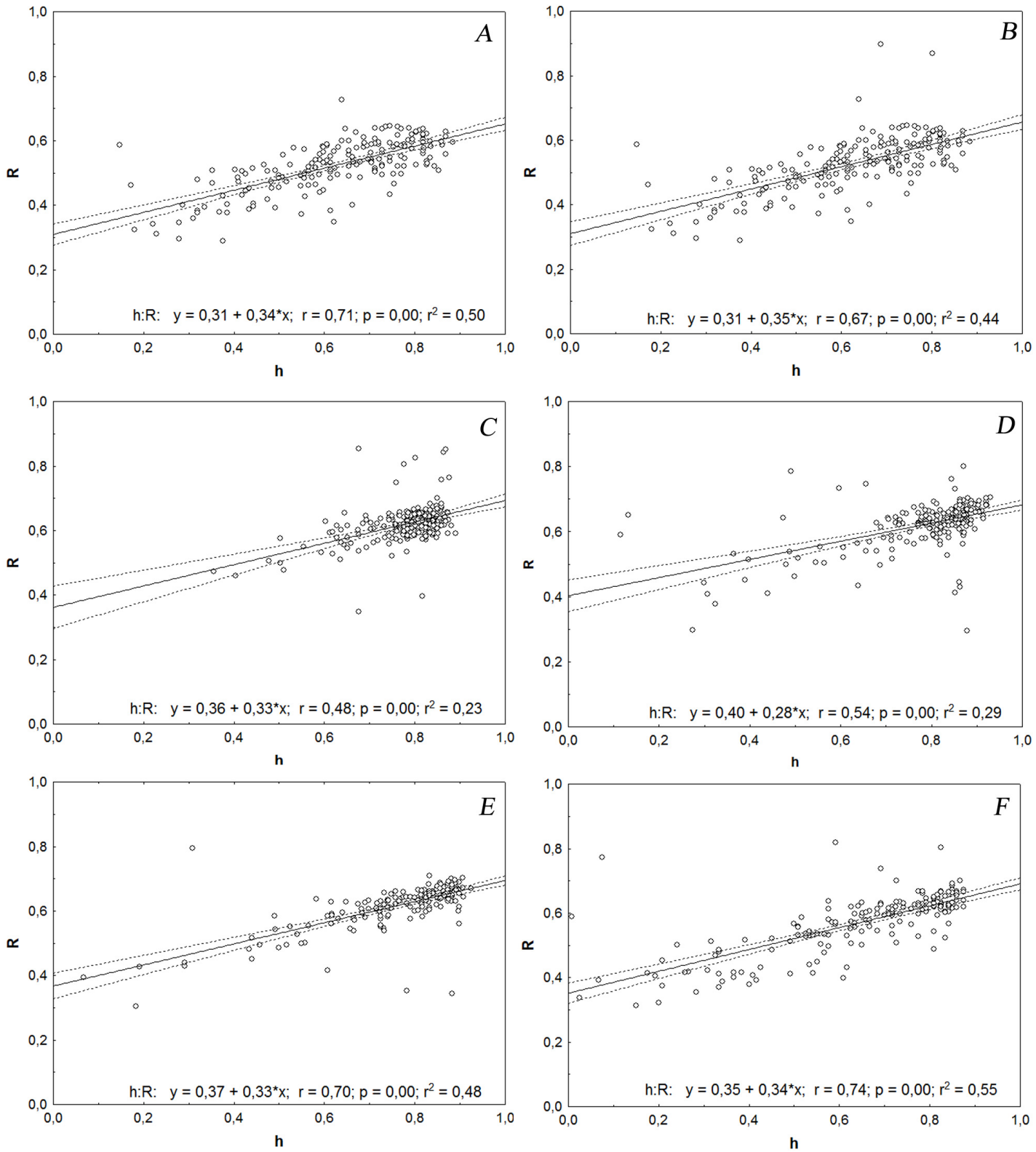

Figura 4 - Relação entre os valores diários de razão de radiação solar global (R) e razão de insolação (h) para os meses de julho (A), agosto (B), setembro (C), outubro (D), novembro (E) e dezembro (F), no período de 2006 a 2012, em Macapá (AP).

meses do período chuvoso estes valores variaram de 0,38 a 0,45 , enquanto nos demais meses do ano oscilaram entre 0,27 a 0,34 . A ocorrência de nebulosidade durante o período chuvoso justifica parcialmente a menor quantidade de insolação, visto que, de acordo com Querino et al (2011), a refletividade de energia solar das nuvens é bem maior do que a refletividade da atmosfera sem nuvens, o que facilita uma maior ou menor transmitância.

Nos meses compreendidos no período chuvoso os coeficientes lineares das retas de regressão foram semelhantes aos propostos por Iqbal (1980), entretanto os valores dos coeficientes angulares mostraram-se abaixo do proposto pelo autor, já que este indica o valor constante de 0,52 para a região de 0 a $60^{\circ}$ de latitude. Desta forma os coeficientes propostos neste trabalho retratam a realidade local por terem sido estimados apenas com dados da região e atualizados.

Quanto aos valores dos coeficientes das retas de regressão no período menos chuvoso, verificou-se que estes diferiram dos propostos por Iqbal (1980), sendo os valores dos coeficientes lineares encontrados neste trabalho superiores aos recomendados por Iqbal (1980) e os angulares, foram em média $60 \%$ inferiores ao proposto pelo referido autor. 
Tabela 3 - Coeficientes linear e angular, coeficientes de correlação de Pearson (r), índices de concordância de Willmott (d), coeficientes de desempenho (c) e classificações para a relação radiação global x insolação de acordo com Camargo e Sentelhas (1997).

\begin{tabular}{lccllll}
\hline \multicolumn{1}{c}{ Mês } & $\begin{array}{c}\text { Coeficiente } \\
\text { linear da } \\
\text { regressão } \\
\text { (a) }\end{array}$ & $\begin{array}{c}\text { Coeficiente } \\
\text { angular da } \\
\text { regressão (b) }\end{array}$ & $\mathbf{r}$ & $\mathbf{D}$ & $\mathbf{c}$ & Classificação \\
\hline Janeiro & 0,28 & 0,43 & 0,82 & 0,85 & 0,66 & Bom \\
\hline Fevereiro & 0,29 & 0,39 & 0,73 & 0,92 & 0,85 & Muito bom \\
Março & 0,26 & 0,43 & 0,76 & 0,89 & 0,73 & Bom \\
Abril & 0,24 & 0,44 & 0,72 & 0,95 & 0,90 & Ótimo \\
Maio & 0,24 & 0,45 & 0,82 & 0,84 & 0,64 & Mediano \\
Junho & 0,26 & 0,43 & 0,75 & 0,63 & 0,30 & Péssimo \\
Julho & 0,31 & 0,34 & 0,71 & 0,99 & 0,77 & Muito bom \\
\hline Agosto & 0,32 & 0,32 & 0,50 & 0,99 & 0,58 & Sofrível \\
Setembro & 0,36 & 0,33 & 0,48 & 1,00 & 0,05 & Péssimo \\
\hline Outubro & 0,40 & 0,28 & 0,54 & & & \\
Novembro & 0,37 & 0,33 & 0,70 & & & Mediano \\
Dezembro & 0,35 & 0,34 & 0,74 & & & Mal \\
\hline Jan-Jun & 0,26 & 0,43 & 0,77 & 0,84 & 0,64 & Bom \\
\hline Jul-Dez & 0,33 & 0,35 & 0,67 & 0,58 & 0,42 & 0,73 \\
\hline Jan-Dez & 0,27 & 0,43 & 0,82 & 0,87 & & \\
\hline
\end{tabular}

$\mathrm{Na}$ análise sazonal (Figura 5), verificou-se que no período chuvoso os valores de radiação solar global e insolação apresentaram-se distribuídos ao longo da reta de regressão, enquanto no período menos chuvoso estes valores se concentraram próximos às maiores abscissas e ordenadas.

Para os coeficientes sazonais, o período chuvoso apresentou coeficientes linear e angular da reta de regressão igual a 0,26 e 0,42 respectivamente, enquanto o período menos chuvoso teve coeficientes iguais a 0,33 e 0,35 . Estes valores indicam que no período chuvoso a transmissividade da atmosfera foi maior (coeficiente "b") em virtude da nebulosidade, ocorrendo o contrário no período menos chuvoso, que apresentou maiores valores do coeficiente "a", indicando que a transmissividade foi menor, entretanto a fração de radiação difusa foi maior devido aos aerossóis presentes na atmosfera. Estes resultados corroboram com os resultados obtidos por Blanco e Sentelhas (2002).

No período menos chuvoso há uma menor taxa de nebulosidade com relação ao chuvoso, e essas condições da atmosfera, que abrangem a presença de vapor d'água, assim como, os constituintes da mesma influenciam significativamente na quantidade de radiação solar que chega à superfície da Terra.

Os valores mais elevados do coeficiente angular da equação proposta por Angström-Prescott se encontram nos meses chuvosos, o que indicou que a radiação solar global difusa foi maior nesse período do ano do que nos meses menos chuvosos, provavelmente devido à nebulosidade e aerossóis presentes na atmosfera. Este fato justifica-se pelos altos valores de temperatura, já que a temperatura máxima chegou a $36^{\circ} \mathrm{C}$, e a velocidade do vento máximo a $3,7 \mathrm{~m} \cdot \mathrm{s}^{-1}$, que favorecem a presença de aerossóis na atmosfera (Figura 6).

$\mathrm{Na}$ Figura 5C, pode-se observar os dados em escala anual, nesta o coeficiente de correlação foi classificado como forte com valor de 0,82 , sendo os coeficientes linear e angular igual a 0,27 e 0,43 , respectivamente, indicando que na região há quantidade significativa de radiação que é transmitida pelas partículas na atmosfera e pouca sofre o processo de difusão.

Entre os dados encontrados por Andrade Júnior et al. (2012) os valores sazonais do coeficiente linear da regressão variaram de $0,31 \pm 0,01$ (período seco) a $0,31 \pm 0,007$ (período chuvoso), e o coeficiente $\mathrm{b}$, de $0,54 \pm 0,01$ (período chuvoso) a $0,51 \pm 0,01$ (período seco). Assim como os valores obtidos conforme Tabela 3 , os coeficientes de a e b pouco divergiram entre si ao longo dos períodos chuvoso e seco, sendo próximos aos valores obtidos a partir da média anual (Figura 5).

Apesar do desempenho aceitável da metodologia de estimativa da radiação solar global proposta pela FAO (Allen et al., 1998), que é amplamente difundida, Carvalho et al. (2011) utilizou-se de estimativas locais de "a" e "b" para estimar a evapotranspiração em Seropédica (RJ). Seus resultados obtidos pelo uso de equações de regressão da radiação, em função da insolação, foram mais satisfatórios do que os estimados pela metodologia proposta pela FAO, quando comparados com os dados de evapotranspiração medidos in loco.

Analisando os valores da Tabela 3, verifica-se que apesar de apresentar moderadas e fortes correlações, os modelos propostos para os meses de junho, agosto, setembro e para o período menos chuvoso não são recomendáveis, de acordo 


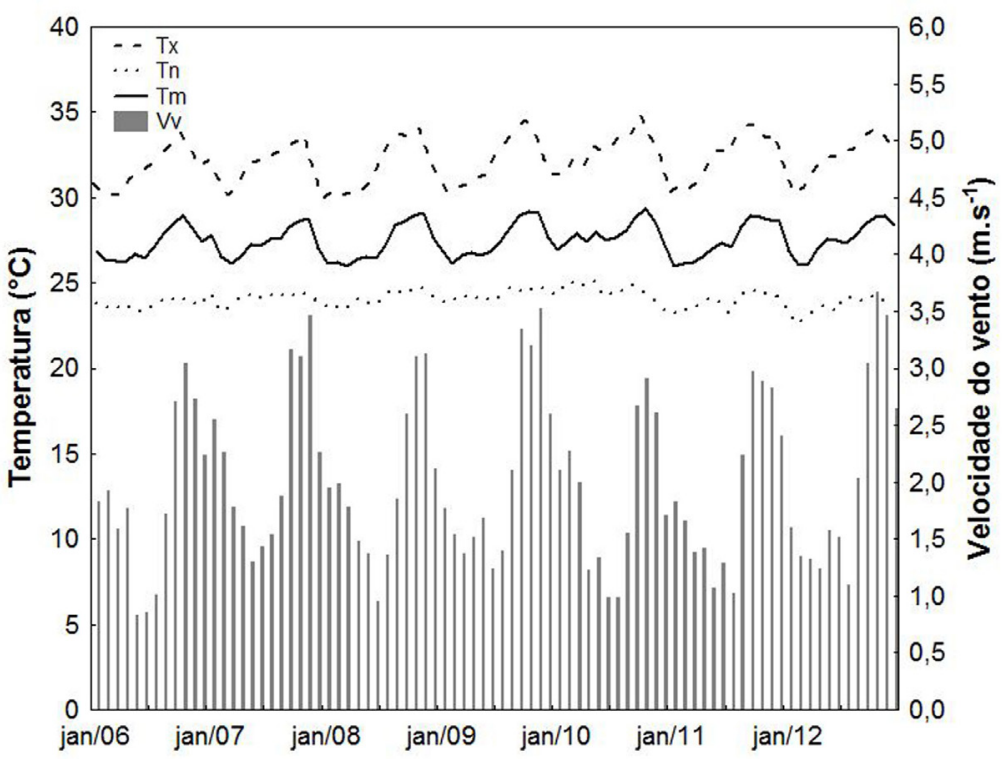

Figura 5 - Variabilidade sazonal da temperatura máxima, média e mínima do ar e da velocidade do vento em Macapá (AP).
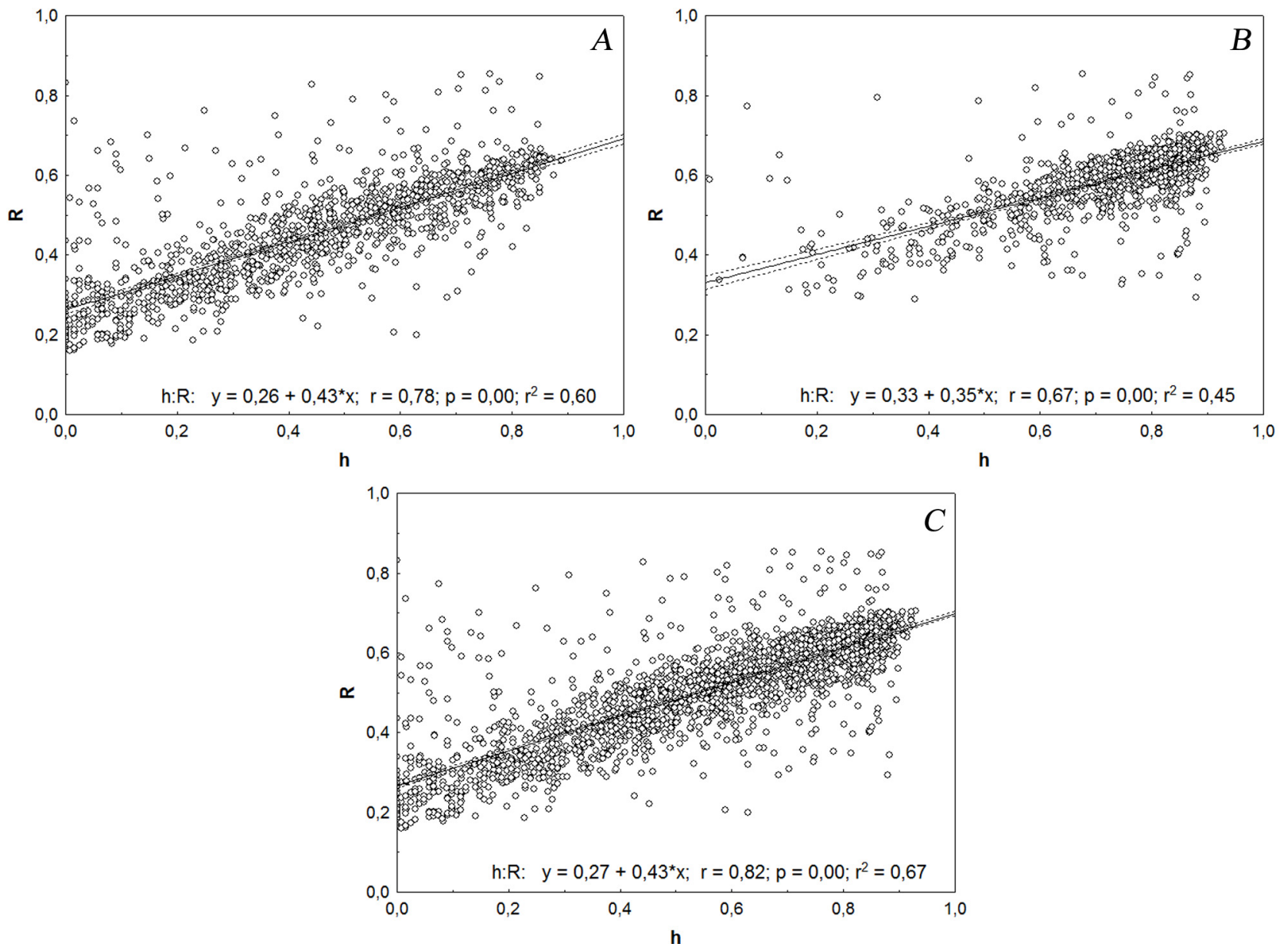

Figura 6 - Relação entre os valores diários de razão de radiação solar global (R) e razão de insolação (h) para o período chuvoso (A), período menos chuvoso (B) e anual (C), de 2006 a 2012, em Macapá (AP). 
com o coeficiente de desempenho, pois apresentaram índices de desempenho inferiores a 0,51. O mau desempenho desses modelos pode ser devido à qualidade dos dados coletados, mesmos estes tendo passado por um teste de qualidade antes do uso neste trabalho ou o modelo não conseguiu representar a variabilidade existente dentro do período analisado, devendo-se, portanto buscar equações com outros ajustes diferentes do linear para realizar-se a estimativa da radiação.

Entretanto, percebe-se que o modelo para dados anuais apresentou bom desempenho sendo aconselhado para o uso de qualquer dia do ano, com índice de desempenho 0,73, revelando que para a estimativa da radiação solar global em Macapá, pode-se usar um único modelo para qualquer mês, este fato deve-se a pouca variabilidade anual da radiação solar na cidade de Macapá.

\section{CONCLUSÕES}

As moderadas e fortes correlações entre os elementos meteorológicos analisados, insolação e radiação solar global, em escala mensal, sazonal e anual evidenciam que a insolação pode ser utilizada para estimar a radiação solar global em Macapá (AP), devendo-se ressaltar que modelos específicos para cada mês devem ser aperfeiçoados, a fim de se obter melhor precisão e eficácia da estimativa.

Os valores dos índices estáticos mostraram que apesar dos modelos propostos apresentarem moderadas e fortes correlações, os meses de junho, agosto, setembro e para o período menos chuvoso apresentaram índices de desempenho fracos. No entanto o modelo anual apresentou bom desempenho sendo aconselhado para o uso de qualquer dia do ano.

\section{REFERÊNCIAS BIBLIOGRÁFICAS}

AGUIAR, L. J. G.; COSTA, J.M. N.; FISCHER, G. R.; AGUIAR, R. G.; DA COSTA, A. C. L.; FERREIRA, W. P. M. Estimativa da radiação de onda longa atmosférica em áreas de floresta $\mathrm{e}$ pastagem no sudoeste da Amazônia. Revista Brasileira de Meteorologia, v. 26, p. 215-224, 2011.

ALLEN, R. G.; PEREIRA, L. S.; RAES, D.; SMITH, M. Crop evapotranspiration: Guidelines for computing crop water requirements: Irrigation and drained, paper 56. Rome: FAO, 1998. 297 p.

ALMOROX, J.; BENITO, M.; HONTORIA, C. Estimation of global solar radiation in Venezuela. Interciencia, v. 33, p. 280-283, 2008.

ANDRADE JÚNIOR, A. S.; NOLETO, D. H.; SILVA, M. E.; BRAGA, D. L.; BASTOS, E. A. Coeficientes da equação de Angstrom-Prescott para Parnaíba, Piauí. Comunicata Scientiae, v. 3, p. 50-54, 2012.
ANGSTRÖN, A. Solar and terrestrial radiation. Quarterly Journal of The Royal Meteorological Society v. 50, p. 121-126, 1924.

ARAUJO, K. D. Variabilidade temporal das condições climáticas sobre as perdas de $\mathrm{CO2}$ na encosta do açude Namorados, em São João do Cariri-PB. 2005. $101 \mathrm{f}$. Dissertação (Mestrado em Manejo e Conservação do Solo e Água) - Centro de Ciências Agrárias, Universidade Federal da Paraíba, Areia, 2005.

BLANCO, F. F.; SENTELHAS, P. C. Coeficientes da equação de Angströn-Prescott para estimativa da insolação para Piracicaba, SP. Revista Brasileira de Agrometeorologia, V. 10, p. 295-300, 2002.

BORGES, V. P.; OLIVEIRA, A. S.; COELHO FILHO, M. A.; SILVA, T. S. M.; PAMPONET, B. M. Avaliação de modelos de estimativa da radiação solar incidente em Cruz das Almas, Bahia. Revista Brasileira de Engenharia Agrícola e Ambiental, v. 14, p. 74-80, 2010.

BURIOL, G. A.; ESTEFANEL, V.; HELDWEIN, A. B.; PRESTES, S.D.; HORN, J. F. C. Estimativa da radiação solar global a partir dos dados de insolação, para Santa Maria - RS. Ciência Rural, v. 42, p. 1563-1567, 2012.

CAMARGO, A. P.; SENTELHAS, P. C. Avaliação do desempenho de diferentes métodos de estimativa da evapotranspiração potencial no Estado de São Paulo, Brasil. Revista Brasileira de Agrometeorologia, v. 5, n. 1, p. 89-97, 1997.

CAMPELO JÚNIOR, J. H. Relação sazonal entre a radiação solar global e insolação no sudoeste da Amazônia. Revista Brasileira de Agrometeorologia, v. 6, p. 193-199, 1998.

CARVALHO, D. F.; SILVA, D. G.; SOUZA, A. P.; GOMES, D. P.; ROCHA, H. S. 2011. Coeficientes da equação de Angstrom-Prescott e sua influência na evapotranspiração de referência em Seropédica, RJ. Revista Brasileira de Engenharia Agrícola e Ambiental, v. 15, p. 838-844, 2011.

CELIK, A. N.; YORUKOGLU, M. A critical review on the estimation of daily global solar radiation from sunshine duration. Energy Conversion and Management, v. 47, p. 2441-2450, 2006.

CULF, A. D.; FISCH, G.; HODNETT, M. G. The albedo of Amazonian forest and ranchland. Journal of Climate, v. 8, p. 1544-1554, 1995.

DANTAS, A. A. A.; CARVALHO, L. G.; FERREIRA, E. Estimativa da radiação solar global para a região de Lavras, MG. Ciência e Agrotecnologia, v. 27, p. 1260-1263, 2003.

DEVORE, J. L. Probabilidade e estatística: para engenharia e ciências. São Paulo, SP: Thomson Pioneira, 2006. 706 p.

DORNELAS, K. D. S.; SILVA, C. L.; OLIVEIRA, C. A. S. Coeficientes médios da equação de Angstrom-Prescott, radiação solar e evapotranspiração de referência em Brasília. 
Pesquisa Agropecuária Brasileira, v. 41, n. 8, p. 12131219, ago. 2006.

GALVÃO, J. A. C.; FISCH, G. Energy budget in forest and pasture sites in Amazonian. Revista Brasileira de Meteorologia, v. 15, p. 25-37, 2000.

HOREL, J. D.; HAHMANN, A. N.; GEISLER, J. E. In investigation of the annual cycle of convective activity over the tropical Americas. Jornal of Climate, v. 2, p. 1388-1403, 1989.

INSTITUTO NACIONAL DE METEOROLOGIA. Normais climatológicas do Brasil 1961-1990. Organizadores: Andrea Malheiros Ramos, Luiz André Rodrigues dos Santos, Lauro Tadeu Guimarães Fortes. Brasília, DF: INMET, 2009. 465 p.

IQBAL, M. An introduction to solar radiation. London: Academic Press, 1980. 390 p.

LIU, X.; MEI, X.; LI, Y.; WANG, Q.; ZHANG, Y.; PORTER, J.R. Variation in reference crop evapotranspiration caused by the Angströn-Prescott coefficient: Locally calibrated versus the FAO recommended. Agricultural Water Management, v. 96, p. 1137-1145, 2009.

MARQUES, D. D.; BRITO, A. U.; CUNHA, A. C.; SOUZA, L. R. Variação da radiação solar no estado do Amapá: Estudo de caso em Macapá, Pacuí, Serra do Navio e Oiapoque no período de 2006 a 2008. Revista Brasileira de Meteorologia, v. 27, p. 127-138, 2012.

MiLleR, D. G.; RIVINGTON, M.; MATTHEWS, K. B.; BUCHAN, K.; BELLOCHI, G. Testing the spatial applicability of the Johnson-Woodward method for estimating solar radiation from sunshine duration data. Agricultural and Forest Meteorology, v. 148, p. 466-480, 2008.
MORADI, I. Quality control of global solar radiation using sunshine duration hours. Energy, v. 34, p. 1-6, 2009.

PEREIRA, S. T.; WELDER ; SILVA, J ; OLIVEIRA, J. S. ; ADENILSON, F.R . Estimativa da radiação solar global para a região de Pedra Azul, norte de Minas Gerais. Enciclopédia Biosfera, v. 6, p. 11-9, 2010.

PERSAUD, N.; LESOLLE, D.; OUATTARA, M. Coefficients of the Angstrom-Prescott equation for estimating global irradiance from hours of bright sunshine in Botswana and Niger. Agricultural and Forest Meteorology, v. 88, p. 27-35.1997.

QUERINO, C. A. S.; MOURA, M. A. L.; QUERINO, J. K. A. S.; VON RADOW, C.; MARQUES FILHO, A. O. Estudo da radiação solar global e do índice de transmissividade (KT), externo e interno, em uma floresta de mangue em Alagoas - Brasil. Revista Brasileira de Meteorologia, v. 26, p. 204-294, 2011.

RIVINGTON, M.; BELLOCHI, G.; MATTHEWS, K. B.; BUCHAN, K. Evaluation of three model estimations of solar radiation at 24 UK stations. Agricultural and Forest Meteorology, v. 132, p. 228-243, 2005.

ROBAA, S. M. Validation of the existing models for estimation global solar radiation over Egypt. Energy Conversion and Management, v. 50, p. 184-193, 2009.

STATSOFT INC. Statistica: data analysis software system, version 9. 2009. CD Rom.

WILLMOTT, C. J.; ACKLESON, S. G.; DAVIS, J. J.; FEDDEMA, K.; KLINK, D. R. Statistics for the evaluation and comparison of models. Journal of Geophysical Research, v. 90, n. 5, p. 8995-9005, 1985. 